\title{
STRICTLY EXTREME AND STRICTLY EXPOSED POINTS
}

\author{
JING HUI QIU \\ Department of Mathematics \\ University of Suzhou \\ Suzhou, Jiang Su Province, China \\ and \\ KELLY MCKENNON \\ Department of Mathematics \\ Washington State University \\ Pullman, Washington, USA \\ (Received February 26, 1990 and in revised form August 8, 1993)
}

\begin{abstract}
The notions of strictly extreme and strictly exposed are introduced. Their properties are discussed, examples are given, and inter-relationships investigated. In particular it is proved that, for separable normed spaces, the strictly extreme points are just the strictly exposed points.
\end{abstract}

KEY WORDS AND PHRASES. Locally convex spaces, extreme points, strictly extreme points, strictly exposed points.

1992 AMS MATH REVIEWS CLASSIFICATION CODE : $52 A 07$.

\section{INTRODUCTION.}

In the sequel, $E$ will denote a Hausdorff, locally convex, real linear space with conjugate space $E^{\prime}$. The closure of a subset $C$ of $E$ will be denoted $C^{-}$, and the convex hull by $[C]$. For two points $\eta$ and $q$ in $E$, we adopt the notation

$$
\begin{aligned}
& |\eta, q| \equiv\{\eta+\lambda(q-\eta): 0<\lambda<1\} \\
& {[\eta, q] \equiv\{n+\lambda(q-\eta): 0 \leq \lambda \leq 1\}}
\end{aligned}
$$

The fundamental definitions and theorems applied in this paper are contained in [1], [2], and [3]. 2. STRICTLY EXTREME POINTS.

Let $C$ be a subset of $E$. The extreme points of $C$ can be characterized in terms of the cone

$$
C(\eta) \equiv\{\lambda v: \lambda>0, \quad v \in E, \quad[\eta, \eta+v] \subset C\} .
$$

An extreme point of $C$ is a point $\eta \in C$ such that $-C(\eta) \cap C(\eta)=\{0\}$. 
DEFINITION 1. A point $\eta$ of a subset $C$ of $E$ is a strictly extreme point of $C$ if $-C(\eta)^{-} \cap$ $C(\eta)^{-}=\{0\}$.

EXAMPLE 1. The strictly extreme points of closed regions bounded by convex polygons are the vertices of the polygons.

EXAMPLE 2. A convex set which is locally uniformly convex has no strictly extreme point. In particular, $L^{p}$-spaces for $1<p<\infty$ have no strictly extreme points in their closed balls.

Other examples will be given below. We presently characterize strictly extreme points in terms of the supporting hyperplanes of $C$. For any point $\eta$ of $C$, let $D(\eta)$ denote the intersection of all closed hyperplanes $H$ through $\eta$ such that $C$ lies completely in either one or the other of the closed half-spaces associated with $H$.

THEOREM 1. Let $\eta$ be a point of a convex subset $C$ of a real locally convex space $E$. A necessary and sufficient condition for $\eta$ to be a strictly extreme point of $C$ is for $D(\eta)$ to be just $\{\eta\}$.

PROOF. We begin by demonstrating

$$
\eta+-C(\eta)^{-} \cap C(\eta)^{-} \subset D(\eta)
$$

Assume false, and consider $x$ not in $D(\eta)$ with $x-\eta \in-C(\eta)^{-} \cap C(\eta)^{-}$. Then there exists a closed hyperplane $H$ supporting $C$ passing through $\eta$ but not $x$. Let $S$ be the corresponding closed half-space containing $C$. Since $x$ is in $\eta+C(\eta)^{-}$, there is a sequence $v_{n}$ in $C(\eta)$ convergent to $x-\eta$. If $x$ were not in $S$, then $\eta+v_{n}$ would eventually be in the (open) complement of $S-$ this however violates the condition that $C$ is within $S$. It follows that $x$ must be in $S$ (but not on its boundary $H)$. Let $\omega_{n}$ be a sequence in $-C(\eta)$ such that $\eta+\omega_{n}$ converges to $x$. Then $\eta+\omega_{n}$ is eventually in $S \backslash H$. Since $\eta$ is in the boundary of $H$ of $S$, it follows that $] \eta-\omega_{n}, \eta[$ is disjoint from $S$. But $\omega_{n}$ is in $-C(\eta)$ and so $] \eta-\omega_{n}, \eta[$ intersects $C$ : absurd.

We now verify the reverse inclusion:

$$
\eta+-C(\eta)^{-} \cap C(\eta)^{-} \supset D(\eta)
$$

Let $x$ be any point in $E$ such that $x-\eta \notin-C(\eta)^{-} \cap C(\eta)^{-}$. Since $-C(\eta)^{-} \cap C(\eta)^{-}$is a closed convex cone, there exists $f \in E^{\prime}$ which is non-negative on $-C(\eta)^{-} \cap C(\eta)^{-}$but is negative at $x-\eta$. If $K$ denotes the kernel of $f$, then $\eta+K$ is a closed supporting hyperplane of $C$, and so $D(\eta) \subset \eta+K$. However $x$ is not in $\eta+K$ since $f(x-\eta)<0$. In particular, $x$ is not in $D(\eta)$, which establishes (2). QED

As a corollary to Theorem 1 we deduce a characterization of strictly extreme points of convex bodies in terms of Minkowski functionals (cf.[4] \$16.4). For a real-valued function $f$ defined on $E$, we denote the left and right Gateaux derivatives of $f$ at $\eta$ in the direction $d$ by $f_{+}^{\prime}(\eta, d)$ and $f_{-}^{\prime}(\eta, d)$ respectively (cf. [4] $\S 26.4$ )

COROLLARY. Let $C$ be a convex subset of $E$ with non-void interior. Let $\eta$ be a boundary point of $C$ and let $q$ be any point in the interior of $C$. Define the Minkowski functional $f$ on $E$ by

(i) $f(x) \equiv \inf \{r>0:(x-q) \in q+r(C-q)\}$ for all $x \in E$. Then a necessary and sufficient condition for $\eta$ to be a strictly extreme point of $C$ is that, for each $x \in E$ not lying on the (unbounded) line determined by $\eta$ and $q$.

(ii) $f_{-}^{\prime}(\eta, x-q)<f_{+}^{\prime}(\eta, x-q)$.

PROOF. Let $F^{-}$and $F^{+}$be the epigraphs of the functions

$$
E \ni x \rightarrow f_{-}^{\prime}(\eta, x-q) \text { and } E \ni x \rightarrow f_{+}^{\prime}(\eta, x-q)
$$


respectively. These are cones $([4] \S 26.4 .7)$ with $F^{+} \subset F^{-}$. There is a theorem to the effect that the points $(y, \varepsilon)$ of $F^{-} \backslash F^{+}$are precisely the points $(y, h(y))$ where $h$ is a continuous affine function whose half-space $\{x \in E: h(x) \leq h(\eta)\}$ contains $C$ ([4] $\{26.4 .11)$. It follows that equation (ii) holds for a point $x \in E$ precisely when $x \notin D(\eta)$. QED

EXAMPLE 3. Let $(X, M, \mu)$ be any measure space. Then the extreme points of the closed unit ball $C$ of the Banach space $E \equiv L^{1}(X, M, \mu)$ are all strictly extreme.

PROOF. Let $\eta$ be an extreme point of $E$. As is well known, $\eta$ must be the $1 \backslash \mu(A)$ times the characteristic function $\varepsilon_{A}$ of some measurable set $A$, no non $\mu$-null subset of which having measure less than $\mu(A)$.

Let $f$ be the gauge of $C$ and $q$ the origin of $E$ : thus

$$
f(x)=\|x\|=\int_{X}|x| d \mu \text { for all } x \in E .
$$

Let $x \in E$ be distinct from the line determined by $\eta$ and $q$ and, for each $\lambda \in \mathbb{R}$ denote by $A(\lambda)$ the set $\{t \in A: \lambda \cdot x(t)<-1 \backslash \mu(A)\}$. We have

$$
f(\eta+\lambda x)-f(\eta)=\int_{A(\lambda)}-2 \backslash \mu(A)-\lambda \cdot x d \mu+\int_{E \backslash A(\lambda)}|\lambda \cdot x| d \mu .
$$

But either $\mu(A(\lambda))=0$ or $\mu(A(\lambda))=\mu(A)$ and, in the latter case, $x$ is almost everywhere equal to some constant value $c$ on $A$. Thus,

$$
f(\eta+\lambda x)-f(\eta)=(-2 \backslash \mu(A)-\lambda \cdot c) \cdot \mu(A(\lambda))+\int_{E \backslash A(\lambda)}|\lambda \cdot x| d \mu .
$$

As $\lambda$ tends to $0, \mu(A(\lambda))$ will eventually be 0 and so

$$
f_{-}^{\prime}(\eta, x-q)=\int_{\{t \in E: x(t)<0\}} x d \mu
$$

and

This implies that

$$
f_{+}^{\prime}(\eta, x-q)=\int_{\{t \in E: x(t)>0\}} x d \mu
$$

$$
f_{-}^{\prime}(\eta, x-q) \leq 0 \leq f_{+}^{\prime}(\eta, x-q) .
$$

At least one of the values $\int_{\{t \in E: z(t)<0\}} x d \mu$ and $\int_{\{t \in E: x(t)>0\}} x d \mathcal{M}$ must be non-zero. It follows from the Corollary to Theorem 1 that $\eta$ is strictly extreme. QED

EXAMPLE 4. Let $(X, M, \mu)$ be any measure space. Then the extreme points of the closed unit ball $C$ of the Banach space $E \equiv L^{\infty}(X, \mathcal{M}, \mu)$ are all strictly extreme.

PROOF. In this proof essup will denote "essential supremum", essinf will denote "essential infimum", and sgn the "signum function".

Let $\eta$ be an extreme point of $E$. As is well-known, $|\eta|$ must equal 1 almost everywhere.

Let $f$ be the gauge of $C$ and $q$ the origin of $E$ : thus

$$
f(x)=\|x\|=\operatorname{essup}|x| \text { for all } x \in E .
$$

Let $x \in E$ be no scalar multiple of $\eta$, and let $\lambda \in \mathbb{R}$ satisfy $|\lambda|<1 \backslash\|x\|$. For almost all $t \in X$ we have

$$
|\eta(t)+\lambda \cdot x(t)|=\operatorname{sgn}(\eta(t)) \cdot(\eta(t)+\lambda \cdot x(t))
$$

whence follows that

$$
f(\eta+\lambda x)-f(\eta)=\operatorname{essup} \operatorname{sgn}(\eta) \cdot \lambda \cdot x
$$


We have

$$
f_{-}^{\prime}(\eta, x-q)=\operatorname{essinf} \operatorname{sgn}(\eta) \cdot x \text { and } f_{-}^{\prime}(\eta, x-q)=\operatorname{essup} \operatorname{sgn}(\eta) \cdot x .
$$

Since $x$ is not a scalar multiple of $\eta$, it follows that $f_{-}^{\prime}(\eta, x-q)$ and $f_{+}^{\prime}(\eta, x-q)$ are distinct. It follows from the corollary to Theorem 1 that $\eta$ is strictly extreme. QED

A convex subset $C$ of a linear space $E$ is said to be polyhedral if the intrinsic core icr(C) of $C$ is nonvoid and the intersection of $C$ with any finite dimensional subspace $F$ of $E$ is a polyhedron of $F$.

EXAMPLE 5. Let the convex subset $C$ of the locally convex space $E$ be polyhedral. Then each extreme point of $C$ is strictly extreme.

PROOF. Assume that $\eta$ is an extreme point of $C$ which is not strictly extreme. Then $-C(\eta)^{-} \cap C(\eta)^{-}$contains at least one straight line $L$ through $O$. Without loss of generality, we may assume that $O \in \operatorname{icr}(\mathrm{C})$. Let $F$ denote the two-dimensional linear subspace generated by $L$ and $\eta$. Then $\eta$ is an extreme point of $C \cap F$ and $L$ is a tangent line of $C \cap F$ at $\eta$ in $F$. It follows that $C \cap F$ is not a polyhedron in $F$ : absurd. QED

The classical Krein-Milman Theorem yields the following:

COROLLARY. Let $C$ be a convex set as in Example 5 above and suppose that $C$ is compact. Then $C$ is the closed convex hull of its strictly extreme points.

\section{STRICTLY EXPOSED POINTS.}

Another sharpening of the idea of an extreme point is that of an exposed point: a point $\eta$ of a subset $C$ of $E$ is exposed if there exists some $f \in E^{\prime}$ for which $f(\eta+x)>f(\eta)$ for all $x \in C(\eta) \backslash\{0\}$. Obviously an exposed point is an extreme point, but the converse is not generally true (consider a boundary point on the juncture of a square surmounted by a half-disk). Since boundary points of a disk are exposed, but not strictly extreme, one might conjecture that the property of being a strictly extreme point is stronger than that of being exposed. Such is not the case however, as can be seen by Example 6 in Section 3 below.

THEOREM 2. Let $\eta$ be a point of a convex subset $C$ of a real locally convex space $E$. A necessary and sufficient condition for $\eta$ to be strictly exposed is for there to exist a function $f \in E^{\prime}$ such that, for any weakly compact subset $K$ of $E \backslash\{0\}$ which intersects $C(\eta)$.

$$
\inf \{f(\eta+x): x \in K \cap C(\eta)\}>f(\eta) \text {. }
$$

PROOF. We first establish the sufficiency of the condition. Since singletons are weakly compact, it is clear that $\eta$ is exposed. If $\eta$ were not strictly exposed there would be a sequence $x_{n}$ in $C(\eta)$ convergent to a point $x_{0} \in C(\eta)^{-} \backslash\{0\}$ such that

$$
\lim _{n \rightarrow \infty} f\left(\eta+x_{n}\right)=f(\eta)
$$

But the union of the singleton $\left\{x_{0}\right\}$ with the range of the sequence $x_{n}$ is weakly compact, so this is not possible.

We now prove the necessity of the condition. Suppose that $\eta$ is strictly exposed and assume that $K$ is weakly compact containing a sequence $x_{n}$ also in $C(\eta)$ such that $\lim _{n \rightarrow \infty} f\left(\eta+x_{n}\right)=f(\eta)$. Then $x_{n}$ admits a subnet $x_{\alpha}$ weakly convergent to a point $x_{0}$ in $K$. Since $C(\eta)$ is convex, its closure coincides with its weak closure and so $x_{0}$ is in $C(\eta)^{-}$, whence follows that $f\left(\eta+x_{0}\right)=f(\eta)$ :absurd. QED 


\section{THE RELATIONSHIP BETWEEN STRICTLY EXTREME AND STRICTLY EXPOSED} POINTS.

That a strictly exposed point is strictly extreme is evident. The following example shows that the converse is not generally true, even in the context of Hilbert spaces.

EXAMPLE 6. Let $E$ be any non-separable Hilbert space and let $B$ be a complete orthonormal subset of $E$. Let $C$ be the set of all $x \in E$ such that $\langle x, b\rangle \geq 0$ for all $b \in B$, and let $\eta$ be the origin of $E$. It is evident that $C=C(\eta)=C(\eta)^{-}$. It is evident as well that $\eta$ is an extreme point of $C$ and so strictly extreme as well. If $f$ were as in the definition of strictly exposed point, then by Riesz's Theorem, there would exist $a \in E$ such that $f(x)=\langle x, a\rangle$ for all $x \in E$. Since $B$ is in $C$, we would have $\langle b, a\rangle>0$ for all $b$ in the uncountable set $B$ : absurd. Hence $\eta$ is not exposed.

In fact $\eta$ is not even exposed when $E$ bears its finest locally convex topology. In this case we would have $f(x)>0$ for all $x \in C$, but $f$ not necessarily corresponding to an element $a$ of $E$ via the inner product. In view of the fact that $B$ is uncountable and equal to the union $\bigcup_{n=1}^{\infty}\{b \in B: f(b) \geq 1 \backslash n\}$, it follows that there is at least one $m \in \mathbb{N}$ such that $\{b \in B: f(b) \geq 1 \backslash m\}$ has a countable infinite subset, say $\left\{b_{n}\right\}_{n=1}^{\infty}$. Let $x$ be the point $\sum_{n=1}^{\infty} b_{n} \backslash n$ in $H$. Since $x-\sum_{i=1}^{n} b_{i} \backslash i$ is in $C$ for each $n \in \mathbb{N}$, we have

$$
f(x) \geq f\left(\sum_{i=1}^{n} b_{i} \backslash i\right) \geq \frac{1}{m} \sum_{i=1}^{n} \frac{1}{i} .
$$

Letting $n$ grow, we see that $f(x)$ would have to be infinite: an absurdity.

THEOREM 3. Let $\eta$ be a point of a convex subset $C$ of a separable normed linear space $E$. The following statements are pairwise equivalent:

(i) $\eta$ is a strictly extreme point;

(ii) $\eta$ is a strictly exposed point.

PROOF. That (ii) implies (i) is trivial, and so we shall deduce (ii) from (i). Let $B$ denote the unit ball relative to the norm on $E$.

Let $y \in C(\eta)^{-} \backslash\{0\}$ be arbitrary. Since $-C(\eta)^{-}$is closed and (i) implies that $y$ is not in this set, there exists $r>0$ such that $-C(\eta)^{-} \cap(y+r B)$ is void. Denote the convex hull $\left[C(\eta)^{-} \cup(y+r B)\right]$ by $K$.

Assume that $-C(\eta) \cap K$ were not void. Then there would be $s, t>0, b \in B$, and $c, d \in C(\eta)^{-}$ such that $-d=t c+s(y+r b)$. But then $y+r b=-(d+t c) \backslash s$ which is in $-C(\eta)^{-}$:absurd. Hence $K$ does not intersect $-C(\eta)$.

Since $K$ has nonvoid interior and does not contain 0 ( 0 being in $-C(\eta)$, it follows that there exists a non-zero element $f_{y}$ of $E^{\prime}$ such that $f_{v}(x) \geq 0$ for all $x \in K$. The intersection of the kernel of $f_{v}$ and the interior of $K$ is evidently void. Since $y$ is in the interior of $K$, we have

$$
f_{y}(y)>0 \text {. }
$$

Let $F$ be the set of all elements of the unit ball of $E^{\prime}$ which are non-negative on $C(\eta)^{-}$. Then $F$ is closed and equicontinuous, thus $\sigma\left(E^{\prime}, E\right)$-compact. Since $E$ is separable, $F$ is $\sigma\left(E^{\prime}, E\right)$ metrizable. It follows that $F$ is $\sigma\left(E^{\prime}, E\right)$-separable and so has a countable dense subset $\left\{f_{n}: n \in \mathbb{N}\right\}$. Let $f$ be the element $\sum_{n=1}^{\infty} f_{n} \backslash 2^{n}$ of $E^{\prime}$. 
Let $y$ be any non-zero element of $C(\eta)^{-}$. Since $f_{v}$ is in $F$, it is in the closure of the sequence $f_{n}$. It follows from (3) that $f_{n}(y)>0$ for some $n \in \mathbb{N}$. Consequently $f(\eta+y)>f(\eta)$, which yields (ii). QED

\section{A RESULT FOR FINITE-DIMENSIONAL SPACES.}

For finite-dimensional spaces, Theorem 3 can be given a somewhat stronger form, which is the purpose of this final section. We begin however with a notion valid for general locally convex spaces.

A strictly exposed point of a subset $C$ of a locally convex space $E$ will be said to the strongly strictly exposed provided that the function $f$ of the definition of strictly exposed may be chosen in such a way that, if $\left\{x_{n}\right\}$ is any sequence in $C(\eta)^{-}$for which $f\left(x_{n}\right)$ converges to 0 , then $x_{n}$ converges to 0 .

LEMMA 1. Let $\eta$ be a strictly extreme point of a convex subset $C$ of a separable normed space $E$. Then there exists a continuous norm \|\| on $E$ such that $\eta$ is strongly strictly exposed relative to this new norm.

PROOF. Let $\left\{f_{n}: n \in \mathbb{N}\right\}$ and $f$ be as in the proof to THEOREM 2. Define \|\| by letting

$$
\|x\| \equiv \sum_{n=1}^{\infty}\left|f_{n}(x)\right| \backslash 2^{n} \text { for all } x \in E .
$$

Evidently we have $f(x)=\|x\|$ for all $x \in C(\eta)$.

It remains only to prove that \|\| is a norm. Suppose that $\|x\|=0$ for some $x \in E$ (which implies that $f_{n}(x)=0$ for each $\left.n \in \mathbb{N}\right)$. Since $C(\eta)$ is a convex cone, its polar $C(\eta)^{\circ}$ is precisely the set of all $h \in E^{\prime}$ such that $h(x) \leq 0$ for all $x \in C(\eta)$. By the duality theory for locally convex spaces, we know that the bi-polar $C(\eta)^{\circ \circ}$ is just $C(\eta)^{-}$. Note that $C(\eta)^{\circ}$ is precisely the set $-F$ (where $F$ is as in the proof to Theorem 2) and recall that $\left\{f_{n}: n \in \mathbb{N}\right\}$ is dense in $F$. It follows that $x$ is in the bi-polar $C(\eta)^{00}=C(\eta)^{-}$. But $C(\eta)^{-}$is just $\{0\}$ by hypothesis. Hence $x$ is the origin and \|\| is a norm. QED

THEOREM 4. Let $\eta$ be a strictly extreme point of a convex subset of a finite dimensional space $E$. Then $\eta$ is strongly strictly exposed.

PROOF. Obviously $E$ is separable and so we may appropriate the norm \|\| of Lemma 1 . Since all Hausdorff locally convex topologies are equivalent for finite dimensional spaces, $\eta$ is strongly strictly exposed. QED

\section{REFERENCES}

1. Diestel, J. and Uhl, J.J. Vector measures, Math. Surveys, No. 15, Amer. Math. Soc., Providence. R.I., 1977.

2. Holmes, R.B. Geometric Functional Analysis and its Applications, Graduate Texts in Mathematics, 24, Springer-Verlag, New York, 1975.

3. Kelley, J.L., Namioka, I. et al. Linear Topological Spaces, Van Nostrand, 1963.

4. Köthe, G. Topological Vector Spaces I, Grndl. Math. Wiss., 159, Springer-Verlag, New York, 1969. 


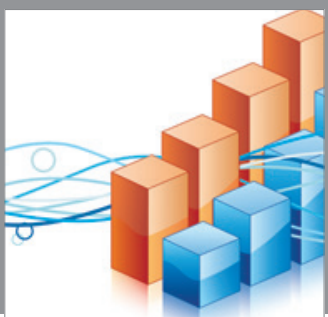

Advances in

Operations Research

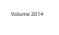

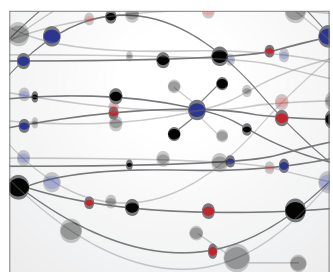

\section{The Scientific} World Journal
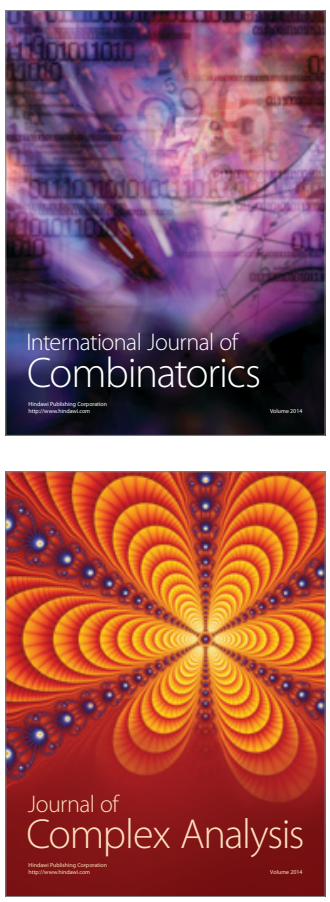

International Journal of

Mathematics and

Mathematical

Sciences
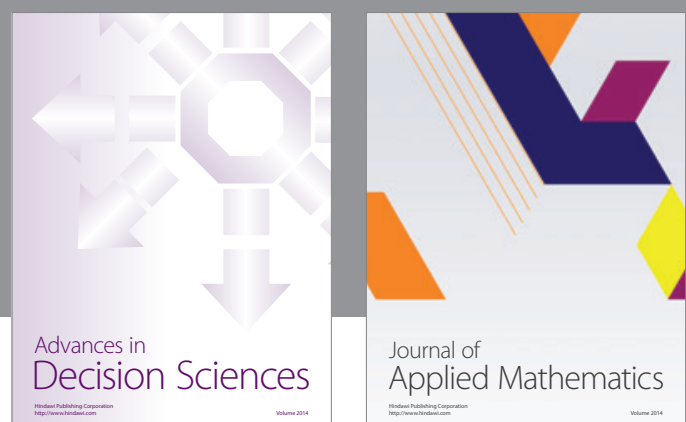

Journal of

Applied Mathematics
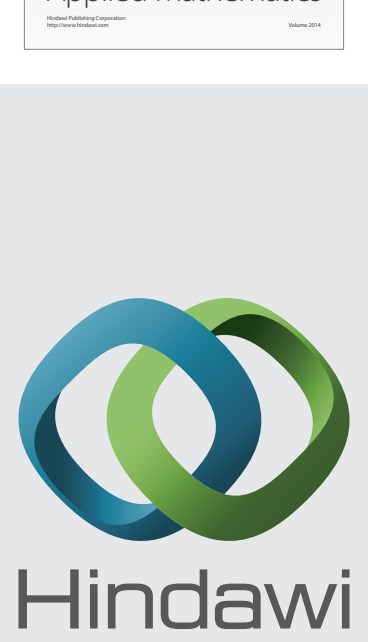

Submit your manuscripts at http://www.hindawi.com
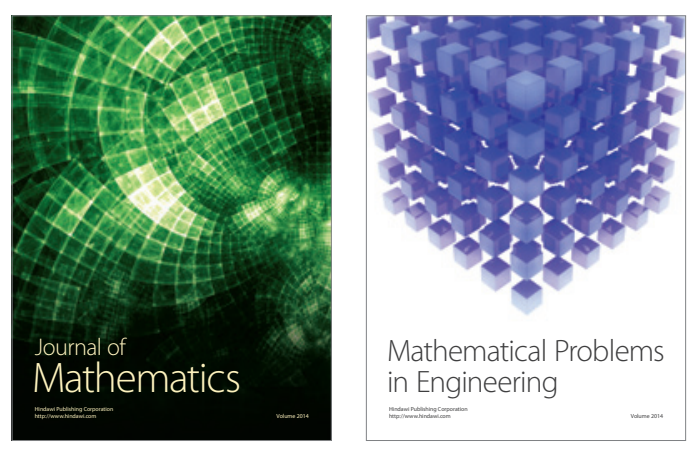

Mathematical Problems in Engineering
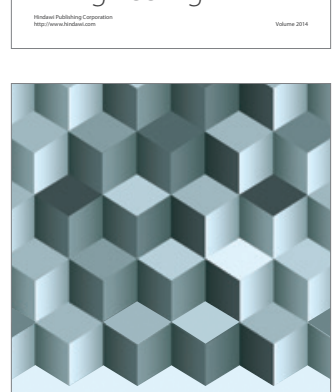

Journal of

Function Spaces
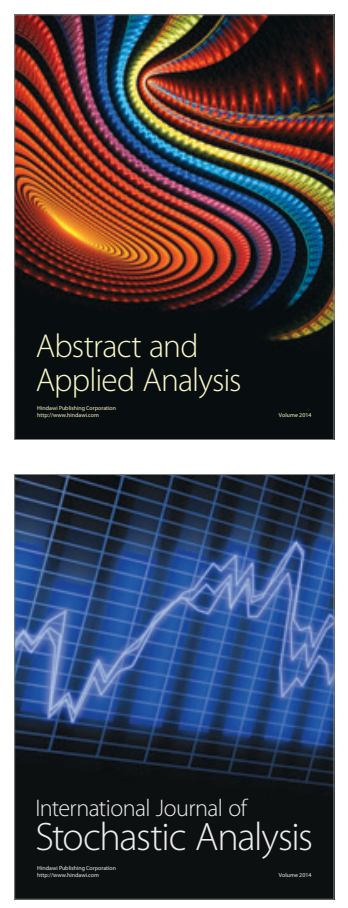

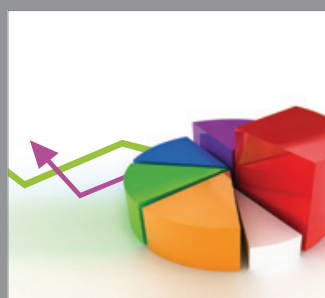

ournal of

Probability and Statistics

Promensencen
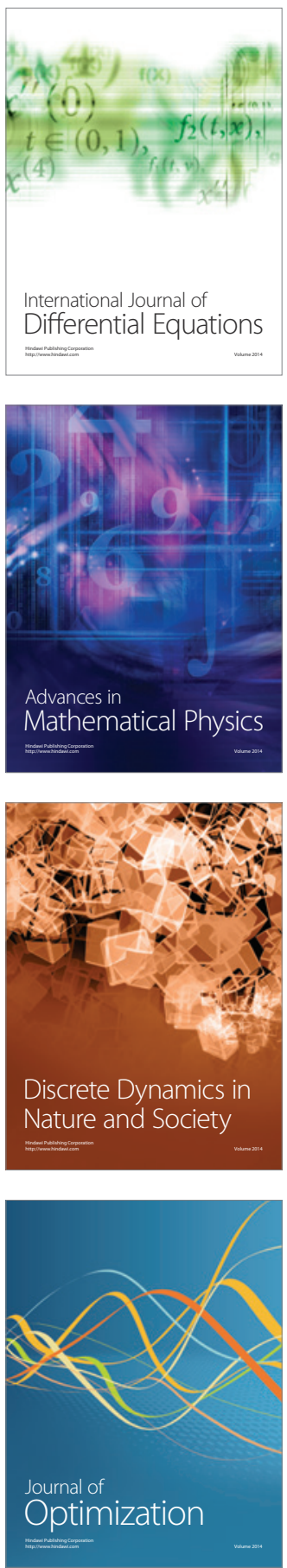